\title{
Digital Morphology Analyzer Sysmex DI-60 vs. Manual Counting for White Blood Cell Differentials in Leukopenic Samples: A Comparative Assessment of Risk and Turnaround Time
}

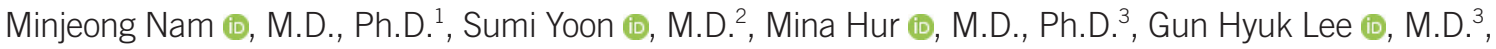

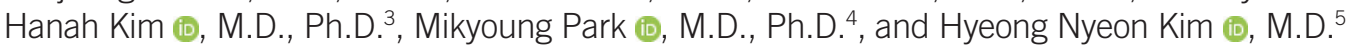 \\ ${ }^{1}$ Department of Laboratory Medicine, Korea University Anam Hospital, Seoul, Korea; ²Department of Laboratory Medicine, Chung-Ang University College of \\ Medicine, Seoul, Korea; ${ }^{3}$ Department of Laboratory Medicine, Konkuk University School of Medicine, Seoul, Korea; ${ }^{4}$ Department of Laboratory Medicine, \\ Eunpyeong St. Mary's Hospital, College of Medicine, The Catholic University of Korea, Seoul, Korea; ${ }^{5}$ Samkwang Medical Laboratories, Seoul, Korea
}

Background: Digital morphology (DM) analyzers are increasingly being used for white blood cell (WBC) differentials. We assessed the laboratory efficiency of the Sysmex DI-60 system (DI-60; Sysmex, Kobe, Japan) in comparison with manual counting in leukopenic samples.

Methods: In total, 40 peripheral blood smear samples were divided into normal, mild leukopenia, moderate leukopenia, and severe leukopenia groups based on WBC count. In each group, the risk and turnaround time (TAT) were compared between DI-60 and manual counting. Risk was determined by failure mode and effect analysis using the risk priority number (RPN) score, and TAT was recorded for the analytical phase.

Results: Overall, DI-60 showed a five-fold lower risk (70 vs. 350 RPN) and longer TAT than manual counting. In severe leukopenic samples, DI-60 showed a shorter TAT/slide and a remarkably lower cell count/slide than manual counting. In all samples, the TAT/cell for DI-60 was substantially longer than that for manual counting (DI-60 vs. manual: total, 1.8 vs. $1.0 \mathrm{sec}$; normal, $1.5 \mathrm{vs}$. $0.7 \mathrm{sec}$; mild leukopenia, $1.9 \mathrm{vs.} 0.9 \mathrm{sec}$; moderate leukopenia, 1.8 vs. $1.0 \mathrm{sec}$; severe leukopenia, 28.8 vs. $19.0 \mathrm{sec}$ ).

Conclusions: This is the first comparative assessment of risk and TAT between DI-60 and manual counting in leukopenic samples. DI-60 decreases the laboratory risk and improves patient safety, but requires more time to count fewer cells, especially in severe leukopenic samples. DM analyzers should be applied selectively depending on the WBC count to optimize laboratory efficiency.

Key Words: Leukopenia, DI-60, WBC differential, Risk, Turnaround time, Laboratory efficiency
Received: September 29, 2021

Revision received: November 10, 2021

Accepted: December 18, 2021

\section{Corresponding author:}

Mina Hur, M.D., Ph.D.

Department of Laboratory Medicine, Konkuk University School of Medicine, Konkuk University Medical Center, 120-1 Neungdong-ro, Gwangjin-gu, Seoul 05030, Korea

Tel: +82-2-2030-5581

E-mail: dearmina@hanmail.net

\section{(c) (i) (8)}

(c) Korean Society for Laboratory Medicine This is an Open Access article distributed under the terms of the Creative Commons Attribution Non-Commercial License (https://creativecommons.org/licenses/by-nc/4.0) which permits unrestricted non-commercial use, distribution, and reproduction in any medium, provided the original work is properly cited.

\section{INTRODUCTION}

White blood cell (WBC) differential is the second most commonly used hematological test to provide crucial clinical information [1]. The gold standard for WBC differential is manual counting of 200 cells each by two experts on a Romanowskystained peripheral blood smear (PBS) [2, 3]. However, the manual method is labor-intensive, time-consuming, and vulnerable to inter- and intra-individual variability and variation in distributed cells among slides [2-5]. 
Several digital morphology (DM) analyzers are used in clinical practice to replace manual counting, and numerous studies have comprehensively evaluated their performance [3, 5-12]. Sysmex DI-60 (DI-60; Sysmex, Kobe, Japan) is the first fully integrated system comprising an XN hematology analyzer, slide maker, staining device, and DM analyzer [10, 11].

In view of laboratory efficiency, risk management, i.e., identifying risk levels and determining priorities for actions to reduce risks, is essential [13]. Reduced risk ensures cost-effectiveness, patient safety and satisfaction, and high-quality laboratory results [14]. For risk management, the Clinical Laboratory Standards Institute (CLSI) guidelines EP23-A theoretically suggest how to assess and manage risks, and the International Organization for Standardization (ISO) 22367:2020 document practically recommends failure mode and effect analysis (FMEA) [15, 16]. Turnaround time (TAT) is another important metric to monitor laboratory efficiency. A short TAT is essential for early diagnosis and treatment, shortened hospital stay, and increased patient satisfaction $[17,18]$. DM analyzers were expected to provide undoubtful advantages over manual counting regarding laboratory efficiency in preventing risks, reducing the workload, simplifying the workflow, and shortening the TAT [19].

Digital images should be validated using different pathological samples in programs for proficiency testing such as the College of American Pathology (CAP) and United Kingdom National External Quality Assurance (UKNEQAS) programs [20, 21]. Thus, most studies have evaluated the performance of DM analyzers using normal and abnormal samples, including different cell types [9-12]. Due to the widespread use of intensive therapies and frequent hematological monitoring, the number of patients with leukopenia has increased [22]. In view of laboratory efficiency, examining slides with leukopenia is more labor-intensive and time-consuming $[3,5,19]$. To the best of our knowledge, no study has evaluated the laboratory efficiency of DM analyzers or focused on leukopenic samples. We hypothesized that the laboratory efficiency of DM analyzers would differ for leukopenic samples. We compared risk and TAT between DI-60 analysis and manual counting using leukopenic samples to assess the laboratory efficiency of both methods.

\section{MATERIALS AND METHODS}

\section{Sample collection}

This in vitro comparative study was conducted between January 2021 and April 2021 at Konkuk University Medical Center (KUMC), Seoul, Korea. The Institutional Review Board of KUMC exempted approval of the study protocol (KUMC 2021-01-041) and waived informed consent. We included 40 samples that were collected for complete blood count (CBC) and PBS. Venous blood samples were collected in $\mathrm{K}_{3}$-EDTA-containing Vacuette ${ }^{\mathrm{TM}}$ tubes (Greiner Bio-One International GmbH, Frickenhausen, Germany), and CBCs were measured using a Sysmex XN-9000 instrument (XN-9000, Sysmex) within four hours of blood collection. Slides were automatically prepared and stained using RAL Wright-Giemsa (RAL Diagnostics, Bordeaux, France) in a Sysmex SP-10 instrument. According to the WBC counts on XN-9000, all samples were divided into four groups: normal, 4.0-10.0 $\times 10^{9} / \mathrm{L}$; mild leukopenia, 2.0-4.0 $\times 10^{9} / \mathrm{L}$; moderate leukopenia, 1.0-2.0 $\times 10^{9} / \mathrm{L}$; and severe leukopenia, $<1.0 \times 10 \% / \mathrm{L}$. In total, 6,921 cells were evaluated using DI-60 analysis and 6,346 cells using manual counting. The sample characteristics (diagnosis and cellular composition) are summarized in Table 1.

WBC differentials using DI-60 analysis and manual counting DI-60 comprises an automated microscope, a high-quality digital camera, and a computer system that collects pre-classified cell images from the slide. Slides prepared using SP-10 were loaded into DI-60. After DI-60 had determined start and end points on the slides for scanning, it scanned them at $10 \times$ magnification in a battlement track pattern. DI-60 acquired cell images at $100 \times$ magnification. For each slide, the instrument was set up with 210 cells as the acquisition cell number, considering acquisition of non-WBCs along with WBCs. Pre-classified cell images were displayed on a computer screen and were verified by the expert. DI-60 provides 18 pre-classified WBC differentials: WBCs, including segmented and band neutrophils, monocytes, lymphocytes, eosinophils, basophils, blast cells, promyelocytes, myelocytes, metamyelocytes, atypical lymphocytes, and plasma cells, and non-WBCs, including smudged cells, artifacts, giant platelets, platelet clumps, erythroblasts, and unidentified cells $[10,11]$. We included only WBCs for the analysis of cell count/slide and TAT/cell.

Manual counting was performed according to the CLSI guidelines H2O-A2 [2]. After slide scanning at low power, two qualified experts counted 200 cells per slide under a light microscope at high power and recorded the results. For leukopenic samples, additional slides should be prepared and cells should be counted according to the CLSI guidelines H2O-A2 [2]. As the purpose of this study was to evaluate risk and TAT in leukopenic samples, not to evaluate the imprecision of WBC counts, we did not evaluate multiple slides per sample. 
Table 1. Sample characteristics

\begin{tabular}{|c|c|c|c|c|c|}
\hline & $\begin{array}{l}\text { Total } \\
(\mathrm{N}=40)\end{array}$ & $\begin{array}{l}\text { Normal } \\
(\mathrm{N}=10)\end{array}$ & $\begin{array}{l}\text { Mild leukopenia } \\
\qquad(\mathrm{N}=10)\end{array}$ & $\begin{array}{l}\text { Moderate leukopenia } \\
\qquad(\mathrm{N}=10)\end{array}$ & $\begin{array}{l}\text { Severe leukopenia } \\
\qquad(N=10)\end{array}$ \\
\hline Age, median (IQR) & $64(33-76)$ & $47(33-71)$ & $77(64-78)$ & $62(62-73)$ & $33(33-65)$ \\
\hline Male, N (\%) & $21(52.5)$ & $6(60)$ & $6(60)$ & $2(20)$ & $7(70)$ \\
\hline \multicolumn{6}{|l|}{ Diagnosis, N (\%) } \\
\hline AML & $18(45)$ & $3(30)$ & $1(10)$ & $7(70)$ & $7(70)$ \\
\hline Lymphoma* & $5(12.5)$ & - & $3(30)$ & $2(20)$ & - \\
\hline PCM & $4(10)$ & $1(10)$ & $1(10)$ & - & $2(20)$ \\
\hline MDS & $3(7.5)$ & - & $3(30)$ & - & - \\
\hline ALL & $1(2.5)$ & - & - & $1(10)$ & - \\
\hline CML & $1(2.5)$ & $1(10)$ & - & - & - \\
\hline $\mathrm{AA}$ & $1(2.5)$ & - & - & - & $1(10)$ \\
\hline No hematologic diseases ${ }^{\dagger}$ & $7(17.5)$ & $5(50)$ & $2(20)$ & - & - \\
\hline WBC counts, median (IQR) & $2,215(610-4,863)$ & $7,010(5,708-7,798)$ & $2,840(2,615-3,400)$ & $1,710(1,125-1,930)$ & $60(50-190)$ \\
\hline \multicolumn{6}{|l|}{ WBC differentials ${ }^{\ddagger}, \mathrm{N}(\%)$} \\
\hline Five differentials & $26(65)$ & $10(100)$ & $2(20)$ & $4(40)$ & $10(100)$ \\
\hline Blasts & $3(7.5)$ & - & $3(30)$ & - & - \\
\hline Immature granulocytes ${ }^{\S}$ & $8(20)$ & - & $5(50)$ & $3(30)$ & - \\
\hline nRBCs & $1(2.5)$ & - & $1(10)$ & - & - \\
\hline Lymphocytes, variant form & $9(22.5)$ & - & $6(60)$ & $3(30)$ & - \\
\hline
\end{tabular}

*Lymphoma included follicular lymphoma $(\mathrm{N}=3)$ and diffuse large $\mathrm{B}$ cell lymphoma $(\mathrm{N}=2) ;{ }^{\dagger}$ No hematologic diseases included bone marrow donors $(\mathrm{N}=2)$,

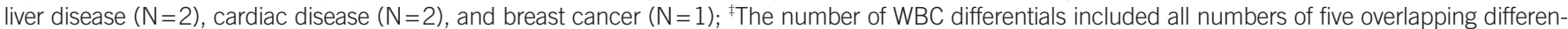
tials, blasts, immature granulocytes, nRBC, and variant lymphocytes; ${ }^{\circledR}$ Immature granulocytes included promyelocytes, myelocytes, and metamyelocytes. Abbreviations: N, number; IQR, interquartile range; AML, acute myeloid leukemia; ALL, acute lymphoblastic leukemia; PCM, plasma cell myeloma; MDS, myelodysplastic syndrome; CML, chronic myeloid leukemia; AA, aplastic anemia; WBC, white blood cell; nRBCs, nucleated red blood cells.

\section{Assessment of risk and TAT}

For risk assessment, FMEA was conducted using the following six steps: (1) determination of the analytical test, (2) assembly of a team, (3) risk identification, (4) risk analysis, (5) risk evaluation, and (6) taking action for risk improvement [13, 23]. In FMEA, DI-60 analysis and manual counting were assessed from placing a slide on the microscope stage to recording of the results. The team consisted of a director, a medical doctor, two qualified medical technicians, and two analyzer specialists. The director, who had experience with FMEA, trained the other team members in identifying potential defects and suggesting potential interventions and preventive actions. All members reviewed each step of DI-60 analysis and manual counting and identified risks, causes, and consequences based on their experience and information in the literature. Before the actual risk assessment, all team members conducted the FMEA independently and then, differences were analyzed and discussed until consensus was reached for objectivity. For each potential risk, scores of severity, occurrence, and detectability were assigned according to the FMEA rating scale (Supplemental Data Table 1). Each score was determined as the median of the scores assigned by the team members. The overall risk priority number (RPN) was calculated by multiplying the three scores. After assessing the risk for each process, all team members suggested preventive actions to reduce the occurrence of risks with high RPN scores.

The TAT was determined for the analytical phases of DI-60 analysis and manual counting, from placing a slide on the microscope stage to recording of the results. For DI-60 analysis, the process of transferring data to a laboratory information system was not instrumentally ready; therefore, we excluded this process from the analytical phases of DI-60 analysis and manual counting. A digital camera recorded the TAT of each process in DI-60 analysis and manual counting. The TAT of DI-60 was additionally checked using a log file of the computer system. Total TAT was calculated as the sum of TATs for all cell counting steps and was represented as the median value (in mins) in each group. The total TAT was transformed into secs for calculating 
Table 2. Comparison of FMEA between DI-60 and manual counting for WBC differentials $(N=40)$

\begin{tabular}{|c|c|c|c|c|c|c|c|c|}
\hline & \multirow{2}{*}{ Process step } & \multirow{2}{*}{ Potential defect } & \multirow{2}{*}{$\begin{array}{l}\text { Potential } \\
\text { intervention }\end{array}$} & \multirow{2}{*}{$\begin{array}{l}\text { Conse- } \\
\text { quence }\end{array}$} & \multicolumn{4}{|c|}{ FMEA } \\
\hline & & & & & S & 0 & D & RPN \\
\hline \multirow{3}{*}{$\begin{array}{l}\text { DI-60 } \\
\text { analysis }\end{array}$} & 1. Insert a slide in the instrument & Wrong slide or mechanical error & Repeat & Delay & 4 & 3 & 1 & 12 \\
\hline & $\begin{array}{l}\text { 3. Acquire images under high power }(100 \times) \\
\text { (pre-classification) }\end{array}$ & Mechanical error & Repeat & Delay & 4 & 1 & 1 & 4 \\
\hline & Total & & & & - & - & - & 70 \\
\hline \multirow{3}{*}{$\begin{array}{l}\text { Manual } \\
\text { counting }\end{array}$} & 1. Place a slide on the microscope stage & Wrong slide or incorrectly labeled slide & Repeat & Delay, WR & 9 & 1 & 6 & 54 \\
\hline & 2. Scan ideal zone under low power $(100 \times)$ & Low quality of slide or broken slide & Re-prepare & Delay & 6 & 2 & 1 & 12 \\
\hline & 3. Count cells under high power $(1,000 \times)$ & Incorrect counting & Repeat & Delay, WR & 9 & 2 & 8 & 144 \\
\hline
\end{tabular}

The RPN scores based on four groups were not calculated due to no difference in the process step.

${ }^{*}$ According to the CLSI guidelines H2O-A2, the manual count was calculated as the mean of the results of two experts. The definite RPN of manual counting was 700 (350×2); we compared only the analytical process between DI-60 and manual counting.

Abbreviations: FMEA, failure mode and effect analysis; WBC, white blood cell; N, number; S, severity; O, occurrence; D, detectability; RPN, risk priority number; WR, wrong result.

Table 3. Comparison of turnaround time between DI-60 and manual counting for WBC differentials

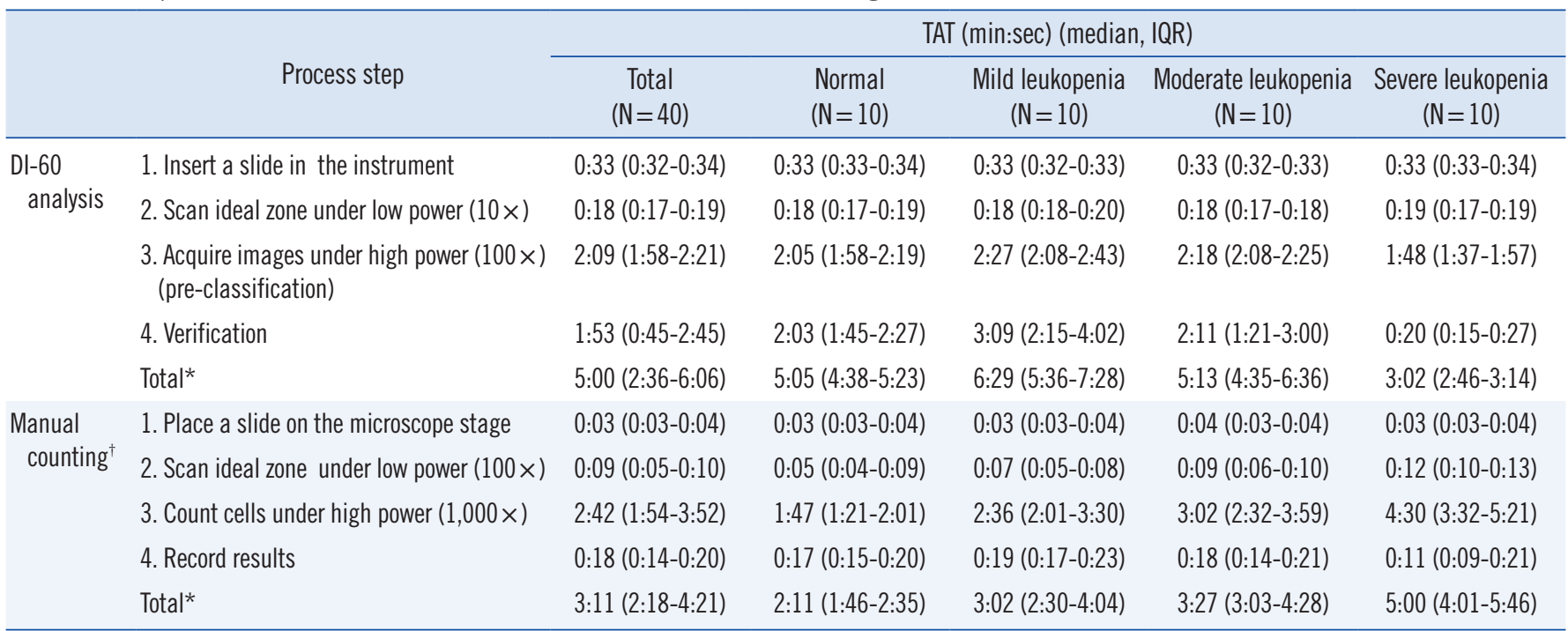

The last process step of data transfer to the laboratory information system was excluded for both DI-60 analysis and manual counting.

* Total value indicates median and IQR of total TAT for counting total cells in each slide in total group and each group; ${ }^{\text {TW }}$ only compared TAT for one analytical process of one expert in manual counting; if the result of manual counting would have been determined as the mean of the results obtained by the two experts, the TAT of manual counting would have been significantly longer.

Abbreviations: WBC, white blood cell; TAT, turnaround time; IQR, interquartile range; N, number.

the overall TAT. TAT/slide represents the overall TAT for cell counting per slide, and TAT/cell represents the overall TAT divided by the total number of counted cells.

All data were analyzed using Microsoft Excel 2016 (Microsoft, Seattle, WA, USA) and MedCalc Statistical Software (v19.4.0, MedCalc Software Bvba, Ostend, Belgium). Normal distribution was checked using the Kolmogorov-Smirnov test, and non-nor- mally distributed data are presented as median and interquartile range.

\section{RESULTS}

DI-60 analysis showed a five-fold lower risk than manual counting (70 vs. 350 RPN) (Table 2). All DI-60 processes showed 
lower severity and detectability than those of manual counting. The RPN for DI-60 pre-classification was remarkably lower than that for manual counting. RPN did not differ among the four WBC count groups (data not shown).

In DI-60 analysis, the TAT for pre-classification and verification showed an inverted-U shape; it increased in mild leukopenia samples, but decreased in severe leukopenia samples. In manual counting, the TAT for cell counting, which was the most timeconsuming process, increased as the number of WBCs decreased. The overall TAT of DI-60 analysis in all groups was longer than that of manual counting, except in severe leukopenia samples (DI-60 vs. manual: total, 5:00 [min:sec] vs. 3:11; normal, 5:05 vs. 2:11; mild leukopenia, $6: 29$ vs. 3:02; moderate leukopenia, 5:13 vs. 3:27; and severe leukopenia, 3:02 vs. 5:00) (Table 3).

In manual counting, the median TAT/slide increased with decreasing number of WBC, whereas in DI-60 analysis, it increased in mild leukopenia samples and decreased in severe leukopenia samples. In severe leukopenia samples, the TAT/slide and total
TAT of DI-60 analysis were both 0.6 -fold those for manual counting (DI-60 vs. manual: 182 vs. 300 seconds; 1,806 vs. 2,951 seconds, respectively) (Fig. 1A). The median cell count/slide in the normal, mild leukopenia, and moderate leukopenia groups was nearly 200 for both DI-60 analysis and manual counting. In severe leukopenia samples, cell count/slide and total cells counted were two-fold and four-fold, respectively, lower for DI-

Table 4. Comparison of TAT/cell between DI-60 analysis and manual counting

\begin{tabular}{lcc}
\hline & \multicolumn{2}{c}{ TAT/cell (sec), (median, IQR) } \\
\cline { 2 - 3 } & DI-60 analysis & Manual counting \\
\hline Total $(\mathrm{N}=40)$ & $1.8(1.5-8.3)$ & $1.0(0.7-2.8)$ \\
Normal $(\mathrm{N}=10)$ & $1.5(1.4-1.6)$ & $0.7(0.5-0.8)$ \\
Mild leukopenia $(\mathrm{N}=10)$ & $1.9(1.6-2.3)$ & $0.9(0.8-1.2)$ \\
Moderate leukopenia $(\mathrm{N}=10)$ & $1.8(1.4-2.3)$ & $1.0(0.9-1.4)$ \\
Severe leukopenia $(\mathrm{N}=10)$ & $28.8(11.8-48.3)$ & $19.0(4.6-40.7)$ \\
\hline
\end{tabular}

Abbreviations: TAT, turnaround time; IQR, interquartile range.

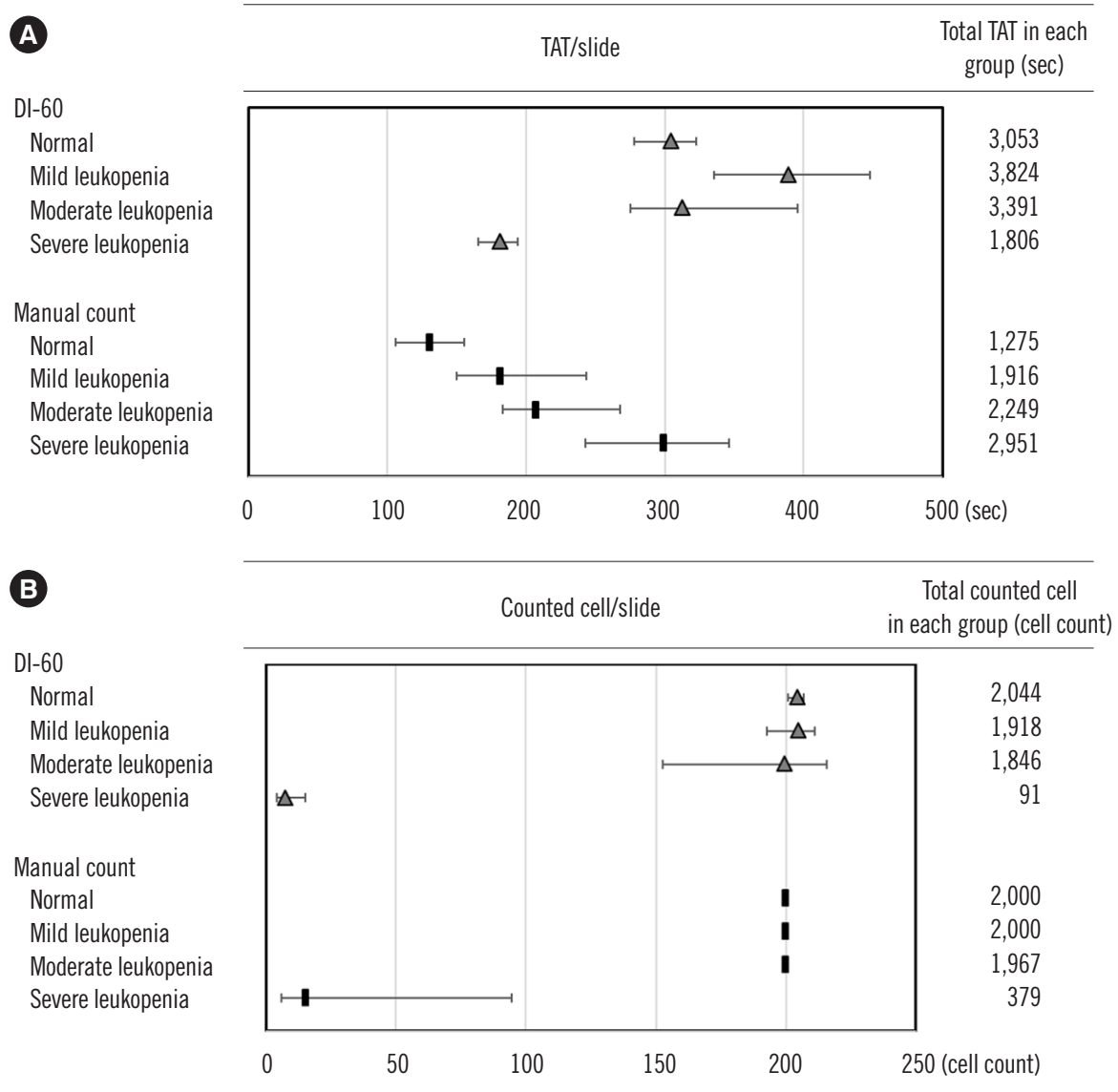

Fig. 1. Comparison of TAT/slide (A) and cell count/slide (B) between DI-60 analysis and manual counting for the four WBC count groups. Triangles and quadrangles indicate median values of DI-60 and manual counting, respectively, and lines indicate interquartile ranges. Total cells counted were summated by each TAT/slide or cell count/slide in each group.

Abbreviations: TAT, turnaround time; WBC, white blood cell. 
60 analysis than for manual counting (DI-60 vs. manual: 8 vs. 16 cells; 91 vs. 379 cells) (Fig. 1B). The median TATs/cell of DI60 analysis for all groups, including severe leukopenia, were longer than those of manual counting (DI-60 vs. manual [seconds]: total, 1.8 vs. 1.0; normal, 1.5 vs. 0.7; mild leukopenia, 1.9 vs. 0.9; moderate leukopenia, 1.8 vs. 1.0; severe leukopenia, 28.8 vs. 19.0) (Table 4).

\section{DISCUSSION}

We compared the risk and TAT between DI-60 analysis and manual counting in leukopenic samples to evaluate the laboratory efficiency of both methods. DI-60 analysis showed a five-fold lower risk than manual counting (70 vs. 350 RPN) (Table 2). All DI-60 processes showed lower severity, detectability, and RPN than the corresponding processes of manual counting. In particular, the RPN of DI-60 pre-classification was remarkably different from that of manual counting. These results indicate that DI-60 can contribute to improved laboratory efficiency and patient safety.

Automated analyzers have been introduced in hematology laboratories and blood bank laboratories; however, few studies have focused on laboratory efficiency in these laboratories with high manual workload [24-26]. These studies demonstrated the usefulness and applicability of FMEA in clinical laboratories and showed that automated methods are associated with lower RPNs than manual methods, which is in line with the present results. The substantially lower RPN of DI-60 analysis reflects that automation reduces or eliminates risk associated with human errors in manual steps. Through regular training and examination, medical technicians can be familiarized with manual steps, which would decrease the occurrence of risks. However, uncertain measurement and the possibility of human error in manual counting remain. Considering the last step of FMEA (taking action for risk improvement), hematology laboratories could use DI-60 analysis rather than manual counting for WBC differentials in leukopenic samples. Alternatively, reducing the RPN in manual counting is possible by decreasing the occurrence or increasing the detectability of prioritized risks with high RPN when the severity cannot be altered [23].

DM analyzers have completely revolutionized hematology laboratories by improving their analytical performance and efficiency, with lower risk and manual workload and shorter TAT [3, 19]. However, our results demonstrated that DI-60 analysis showed a longer TAT than manual counting in leukopenic samples (Table 3). Regardless of the WBC count, the TAT of slide insertion and ideal zone scanning in DI-60 analysis and that of slide insertion, ideal zone scanning, and result recording in manual counting were not considerably different and were relatively short. However, mild leukopenic samples showed the longest TAT for pre-classification and verification in DI-60 analysis, whereas severe leukopenic samples showed the shortest. The cellular composition of mild leukopenic samples included five differentials, blasts, immature granulocytes, nucleated red blood cells (nRBCs), and variant lymphocytes, and that of severe leukopenic samples simply included five differentials. Thus, for mild leukopenic samples with diverse cell types, the TAT was relatively long, whereas severe leukopenic samples with a simple cellular composition required less time for pre-classification and verification in DI-60 analysis. In manual counting, as the number of WBC decreased, the TAT of cell counting remarkably increased, likely because it requires substantially more time to search for cells to count on slides of severe leukopenic samples than on those of samples with a normal WBC count.

Although the CLSI guidelines H2O-A2 recommend counting at least 200 cells, this is not always feasible, especially in case of severe leukopenia [2]. The median cell count/slide in normal, mild leukopenia, and moderate leukopenia groups was nearly 200 for both DI-60 analysis and manual counting, but this number was not achievable in cases of severe leukopenia. The short TAT of DI-60 analysis for severe leukopenia samples implies that DI-60 does not actually spend less time on cell counting, but counts fewer cells than are counted in the manual method. Moreover, in manual counting, the experts discriminated between WBCs and non-WBCs, and the latter were not counted. However, DI-60 acquired images before starting the analysis and pre-classified the cells into WBCs and non-WBCs (e.g., a total of 6,921 cells $=5,899$ WBCs+1,022 non-WBCs). Non-WBCs, including smudge cells, artifacts, and unidentified cells, should have been eliminated during verification. Thus, in severe leukopenia samples, DI-60 spends substantially more time on cell counting (Table 4).

This study had some limitations. TAT can be defined in various ways based on test, priority (emergency or routine), patient (inpatient, outpatient, or emergency department patient), and activity (time of ordering or sample receipt in the laboratory), and a standard protocol to measure TAT is lacking [27]. Although laboratory testing includes pre-analytical, analytical, and postanalytical phases, we only assessed the TAT of the analytical phase [28]. The pre-analytical and post-analytical phases were difficult to control for TAT measurement due to diverse external factors. If the definition of TAT or protocol to measure TAT was 
different, TAT comparison between DI-60 analysis and manual counting might have led to a different conclusion. In addition, although the samples with mild and moderate leukopenia contained various pathologic cell types, we compared the risk and TAT rather specifically in leukopenic samples, not in broad pathologic samples. A further comparison of risk and TAT between DI-60 analysis and manual counting would require using more pathologic samples with mixed cellular composition. For efficient utilization of DI-60, further comparative studies to verify the accuracy and precision of WBC count in leukopenic samples are also warranted [29]. Finally, our sample number was rather small; however, we counted 6,921 cells using DI-60 and 6,346 cells by manual counting, which is sufficient to fulfill our study purpose of comparing risk and TAT between DI-60 and manual counting.

In conclusion, this was the first study comparing risk and TAT between DI-60 analysis and manual counting in leukopenic samples. We demonstrated that DI-60 substantially reduces risks in the analytical phase, but spends more time on counting fewer cells in severe leukopenic samples than manual counting. Based on the current need for improved laboratory efficiency, the implementation of DM analyzers is considered inevitable, especially in high-volume laboratories with a large burden of pathologic or leukopenic samples. However, our results underscore that DM analyzers do not necessarily ensure high laboratory efficiency and may even prolong TAT. To optimize laboratory efficiency, DM analyzers should be applied selectively depending on the sample type and each laboratory's situation and only after risk and TAT assessment.

\section{ACKNOWLEDGEMENTS}

None.

\section{AUTHOR CONTRIBUTIONS}

Nam $\mathrm{M}$ analyzed the data and wrote the draft; Hur M designed the study and finalized the draft; Yoon S, Park M, and Kim HN participated in data collection; Lee $\mathrm{GH}$ and Kim $\mathrm{H}$ reviewed the draft. All authors have accepted responsibility for the entire content of this manuscript and approved the final version.

\section{CONFLICTS OF INTEREST}

The authors report no conflicts of interest. The authors alone are responsible for the content and writing of the paper.

\section{RESEARCH FUNDING}

This work was supported by a Konkuk University Medical Center Research Grant 2021 (grant no. K210105).

\section{ORCID}

Minjeong Nam

https://orcid.org/0000-0003-3542-3487

Sumi Yoon

Mina Hur

Gun Hyuk Lee

Hanah Kim

Mikyoung Park

Hyeong Nyeon Kim https://orcid.org/0000-0002-4429-9978 https://orcid.org/0000-0002-9278-4572 https://orcid.org/0000-0002-3266-638X https://orcid.org/0000-0002-4815-6958 https://orcid.org/0000-0003-3423-8909

\section{REFERENCES}

1. Stouten K, Riedl JA, Levin MD, van Gelder W. Examination of peripheral blood smears: performance evaluation of a digital microscope system using a large-scale leukocyte database. Int J Lab Hematol 2015;37:e13740.

2. CLSI. Reference leukocytes (WBC) differential count (proportional) and evaluation of instrumental methods; approval guideline. 2nd ed. CLSI Document H20-A2. Wayne, PA: Clinical Laboratory Standards Institute, 2007.

3. Kratz A, Lee SH, Zini G, Riedl JA, Hur M, Machin S, International Council for Standardization in Haematology. Digital morphology analyzers in hematology: ICSH review and recommendations. Int J Lab Hematol 2019; 41:437-47

4. Novis DA, Walsh M, Wilkinson D, St Louis M, Ben-Ezra J. Laboratory productivity and the rate of manual peripheral blood smear review: a College of American Pathologists Q-Probes study of 95,141 complete blood count determinations performed in 263 institutions. Arch Patho Lab Med 2006;130:596-601.

5. Kratz A, Lee SH, Zini G, Hur M, Machin S. Rebuttal of a paper submitted by Hans-Inge Bengtsson Comment. Int J Lab Hematol 2020;42: e216-7.

6. Tatsumi N and Pierre RV. Automated image processing. Past, present, and future of blood cell morphology identification. Clin Lab Med 2002; 22:299-315, viii.

7. Kratz A, Bengtsson HI, Casey JE, Keefe JM, Beatrice GH, Grzybek DY, et al. Performance evaluation of the CellaVision DM96 system: WBC differentials by automated digital image analysis supported by an artificial neural network. Am J Clin Pathol 2005;124:770-81.

8. Cornet E, Perol JP, Troussard X. Performance evaluation and relevance of the CellaVision DM96 system in routine analysis and in patients with malignant hematological diseases. Int J Lab Hematol 2008;30:536-42.

9. Park SH, Park CJ, Choi MO, Kim MJ, Cho YU, Jang S, et al. Automated digital cell morphology identification system (CellaVision DM96) is very useful for leukocyte differentials in specimens with qualitative or quantitative abnormalities. Int J Lab Hematol 2013;35:517-27.

10. Tabe Y, Yamamoto T, Maenou I, Nakai R, Idei M, Horii T, et al. Performance evaluation of the digital cell imaging analyzer DI-60 integrated into the fully automated Sysmex XN hematology analyzer system. Clin Chem Lab Med 2015;53:281-9. 
11. Kim HN, Hur M, Kim H, Kim SW, Moon HW, Yun YM. Performance of automated digital cell imaging analyzer Sysmex DI-60. Clin Chem Lab Med 2017;56:94-102.

12. Yoon S, Hur M, Park M, Kim H, Kim SW, Lee TH, et al. Performance of digital morphology analyzer Vision Pro on white blood cell differentials. Clin Chem Lab Med 2021;59:1099-106.

13. Njoroge SW and Nichols JH. Risk management in the clinical laboratory. Ann Lab Med 2014;34:274-8.

14. Sciacovelli L, Lippi G, Sumarac Z, West J, Garcia Del Pino Castro I, Furtado Vieira K, et al. Quality indicators in laboratory medicine: the status of the progress of IFCC working group "Laboratory errors and patient safety" project. Clin Chem Lab Med 2017;55:348-57.

15. Medical laboratories-application of risk management to medical laboratories. International Organization for Standardization (ISO) 22367. 2020.

16. CLSI. Laboratory quality control based on risk management; approved guideline. 2nd ed. CLSI document EP23-A. Wayne, PA: Clinical and Laboratory Standards Institute, 2011.

17. Holland LL, Smith LL, Blick KE. Reducing laboratory turnaround time outliers can reduce emergency department patient length of stay: an 11-hospital study. Am J Clin Pathol 2005;124:672-4.

18. Dolci A, Giavarina D, Pasqualetti S, Szőke D, Panteghini M. Total laboratory automation: do stat tests still matter? Clin Biochem 2017;50:60511.

19. Lippi G and Da Rin G. Advantages and limitations of total laboratory automation: a personal overview. Clin Chem Lab Med 2019;57:802-11.

20. Pantanowitz L, Sinard JH, Henricks WH, Fatheree LA, Carter AB, Contis $L$, et al. Validating whole slide imaging for diagnostic purposes in pathology: guideline from the College of American Pathologists pathology and laboratory quality center. Arch Pathol Lab Med 2013;137:1710-22.

21. Rosetti M, Massari E, Poletti G, Dorizzi RM. Could the UKNEQAS program "Manual differential blood count" be performed by the use of an automated digital morphology analyzer (Sysmex DI-60)? A feasibility study. Clin Chem Lab Med 2021;59:e161-4.

22. Saloustros E, Tryfonidis K, Georgoulias V. Prophylactic and therapeutic strategies in chemotherapy-induced neutropenia. Expert Opin Pharmacother 2011;12:851-63.

23. Jiang $Y$, Jiang $H$, Ding $S$, Liu $Q$. Application of failure mode and effects analysis in a clinical chemistry laboratory. Clin Chim Acta 2015;448:805.

24. Han TH, Kim MJ, Kim S, Kim HO, Lee MA, Choi JS, et al. The role of failure modes and effects analysis in showing the benefits of automation in the blood bank. Transfusion 2013;53:1077-82.

25. Chung HJ, Hur M, Choi SG, Lee HK, Lee S, Kim H, et al. Benefits of VISION Max automated cross-matching in comparison with manual crossmatching: a multidimensional analysis. PLoS One 2019;14:e0226477.

26. Chinudomwong P, Khongjaroensakun N, Chatachote B, Chaothai N, Paisooksantivatana K. Improving the efficiency of the autoverification workflow for nucleated red blood cell reporting in the hematology laboratory. Int J Lab Hematol 2021;43:1373-8.

27. Hawkins RC. Laboratory turnaround time. Clin Biochem Rev 2007;28: 179-94.

28. Lundberg GD. Acting on significant laboratory results. JAMA 1981;245: 1762-3.

29. Yoon S, Hur M, Lee GH, Nam M, Kim H. How reproducible is the data from Sysmex DI-60 in leukopenic samples? Diagnostics 2021;11;2173 
Supplemental Data Table S1. Rating scale of severity, occurrence, and detectability of risk to calculate the risk priority number in failure mode and effect analysis

\begin{tabular}{|c|c|c|c|}
\hline Score & Severity & Occurrence & Detectability \\
\hline 1 & No effect & Remote & $\begin{array}{l}100 \% \text { instrumental detection with preventive } \\
\text { maintenance }\end{array}$ \\
\hline 2 & Very minor interference & & $\begin{array}{l}100 \% \text { instrumental detection with preventive } \\
\text { maintenance }\end{array}$ \\
\hline 3 & Minor interference & Low/relatively few & $\begin{array}{l}100 \% \text { instrumental detection with preventive } \\
\text { maintenance }\end{array}$ \\
\hline 4 & Creates some rework/very low interference & & Partial detection by medical technicians \\
\hline 5 & Creates moderate rework/low interference & Once a week & Periodic detection by medical technicians \\
\hline 6 & Creates considerable rework/moderate interference & & $100 \%$ manual detection and provides visual cues \\
\hline 7 & Causes considerable instrument downtime rework/high interference & & $100 \%$ manual detection \\
\hline 8 & $\begin{array}{l}\text { Could be reportable error and possible instrument downtime/very high } \\
\text { interference }\end{array}$ & Once a day & Low chance to detect with random manual inspection \\
\hline 9 & $\begin{array}{l}\text { Could harm patient but not death and/or possible instrument failure/ } \\
\text { serious interference }\end{array}$ & & $\begin{array}{l}\text { Remote chance to detect with random manual } \\
\text { inspection }\end{array}$ \\
\hline 10 & $\begin{array}{l}\text { Could injure a patient or cause death and/or cause possible instrument } \\
\text { failure/hazardous interference }\end{array}$ & More than once a day & No detection or inspection \\
\hline
\end{tabular}

This table was created referring to ISO 22367:2020 [15], Han, et al. [24], and Chung, et al. [25]. 